\title{
Two Dimensional Adaptive Filter Using Laguerre Function
}

\author{
Chien-Cheng Tseng and Soo-Chang Pei \\ Department of Electrical Engineering \\ National Taiwan University \\ Taipei, Taiwan, R.O.C.
}

\begin{abstract}
In this paper, a new two dimensional (2D) adaptive filter structure is developed. It is based on modeling the impulse response of the 2D IIR systems as a finite linear combination of a set of 2D Laguerre orthonormal functions. When long impulse response system is required to identify, computer simulation shows that the 2D Laguerre filter has better performance than the conventional 2D FIR filter at the same computation cost.
\end{abstract}

\section{Introduction}

Adaptive IIR filtering has recently drawn great interest among researchers in the adaptive signal processing community [1]. This is due to its potential of achieving better performance and more computationally efficient than adaptive FIR filters. Despite such a relative success, FIR systems are almost used exclusively. This is due to the difficulty of ensuring stability and the existence of local minima in the error surface of IIR system.

In order to obtain simultaneously desireable features of both IIR and FIR system: trivial stability and computational efficiency, many researchers have used orthogonal functions such as Laguerre, Legendre polynomials to approximate IIR systems [2]-[4]. In system identification and echo cancelling, these ideas have been shown very successful. However, all adaptive algorithms are restricted to $1 \mathrm{D}$ filter case. The $2 \mathrm{D}$ adaptive algorithm is still not investigated.

In this paper, we derive a new $2 \mathrm{D}$ adaptive algorithm using 2D Laguerre sequence. This filter has the desirable stability, unimodal error surface, and modular implementation. When 2D Laguerre filter is required to identify $2 \mathrm{D}$ system with long impulse response, computation simulation shows that it has better performance than the conventional 2D LMS FIR filter [5].

\section{2D Adaptive Laguerre Filter}

\subsection{D Laguerre functions:}

In the following, the 1D Laguerre function is first reviewed. Then we extend it to $2 \mathrm{D}$ function case.

Fact 1: Let the $\mathrm{Z}$ transform of 1D Laguerre sequence $l_{k}(n, b)$ denoted by

$$
L_{k}(z, b)=\sum_{n=0}^{\infty} l_{k}(n, b) z^{-n}=\sqrt{1-b^{2}} \frac{\left(z^{-1}-b\right)^{k}}{\left(1-b z^{-1}\right)^{k+1}}
$$

then it can be shown that

$$
<l_{k 1}(n, b), l_{k 2}(n, b)>=\delta(k 1-k 2)
$$

where $0<b<1$ and $\delta($.$) is delta function.$

Fact 2: Let the $\mathrm{Z}$ transform of $2 \mathrm{D}$ Laguerre sequence $l_{k 1 k 2}(m, n)$ denoted by

$$
\begin{aligned}
L_{k 1 k 2}(z 1, z 2) & =\sum_{m=0}^{\infty} \sum_{n=0}^{\infty} l_{k 1 k 2}(m, n) z 1^{-m} z 2^{-n} \\
& =L_{k 1}(z 1, b 1) L_{k 2}(z 2, b 2)
\end{aligned}
$$

then it can be shown that

$$
<l_{k 1 k 2}(m, n), l_{k 3 k 4}(m, n)>=\delta(k 1-k 3) \delta(k 2-k 4)
$$

where $0<b 1<1$ and $0<b 2<1$.

2.2 Filter Structure

The input-output relation of $2 \mathrm{D}$ linear time invariant (LTI) causal system is given by the convolution sum 


$$
y(m, n)=\sum_{i=0}^{\infty} \sum_{j=0}^{\infty} h(i, j) x(m-i, n-j)
$$

Now, impulse response $h(m, n)$ is expanded in term of 2D Laguerre orthonormal sequence $\left\{l_{k 1 k 2}(m, n)\right\}$ :

$$
h(m, n)=\sum_{k 1=0}^{\infty} \sum_{k 2=0}^{\infty} w_{k 1 k 2} l_{k 1 k 2}(m, n)
$$

(6) The following formula reflects the tradeoff of resolution versus memory area for fixed $M$ and $N$ :

$$
w_{k 1 k 2}=<h(m, n), l_{k 1 k 2}(m, n)>
$$

Substitute eq(6) into eq(5) and change the order of summation, we get

$$
y(m, n)=\sum_{k 1=0}^{\infty} \sum_{k 2=0}^{\infty} w_{k 1 k 2} v_{k 1 k 2}(m, n)
$$

where

$$
v_{k 1 k 2}(m, n)=\sum_{i=0}^{\infty} \sum_{j=0}^{\infty} l_{k 1 k 2}(i, j) x(m-i, n-j)
$$

In practice, only a finite number of Laguerre sequences can be used. As such, eq(8) is trunced into following finite summation terms:

$$
y(m, n)=\sum_{k 1=0}^{M} \sum_{k 2=0}^{N} w_{k 1 k 2} v_{k 1 k 2}(m, n)
$$

Thus, we can implement 2D Laguerre filter by the structure shown in Fig.1. When $b 1=0$ and $b 2=0$, this filter reduces to the 2D FIR filter. For $b 1, b 2 \neq 0$, this filter is IIR type and its stability is guaranteed if $0<\left|b_{1}\right|<1$ and $0<\left|b_{2}\right|<1$.

\subsection{Memory Area Versus Filter Order}

Memory depth concept has been used to study the performance of $1 \mathrm{D}$ gamma filter [6]. Here, we introduce similar memory area (MEMA) concept to discuss the properties of 2D Laguerre filter. The memory area $A E M A$ for the $M \mathrm{x} N$ th order Laguerre filter is defined as

$$
\begin{aligned}
M E M A & =\sum_{m=0}^{\infty} \sum_{n=0}^{\infty} m n l_{M N}(m, n) \\
& =\left(\alpha_{1}+\beta_{1} M\right)\left(\alpha_{2}+\beta_{2} N\right)
\end{aligned}
$$

where

$$
\begin{aligned}
\alpha_{i} & =\frac{b_{i} \sqrt{1-b_{i}^{2}}}{\left(1-b_{i}\right)^{2}} \\
\beta_{i} & =\frac{1+b_{i} \sqrt{1-b_{i}^{2}}}{\left(1-b_{i}\right)^{2}} \quad i \neq 1,2
\end{aligned}
$$

Next we define the spatial resolution $R$ of the filter as the number of free parameters per unit of area in the filter memory. This is equivalent to the number of free parameters $(M N)$ divided by the memory area $M E M A$ :

$$
R=\frac{M N}{M E M A}
$$

$$
M N=M E M A \times R
$$

Such a tradeoff is impossible in 2D FIR filter, since the fixed choice of $b 1=0$ and $b 2=0$ set the memory area and resolution to $M E M A=M N$ and $R=1$. However, in 2D Laguerre filter, the memory area and resolution can be adapted by variation of $b 1$ and $b 2$.

As an example, assume a signal $y(m, n)$ whose dynamics is described by a system with 2 parameters and maximum delay $5 \times 10$ :

$y(m, n)=0.6 x(m-1, n-2)+0.5 x(m-5, n-10)$

If we try to model this signal with a FIR filter, the order choice $M=5, N=10$ leads to overfitting. However, $M<5, N<10$ leaves the filter unable to incorporate the influence of $x(m-5, n-10)$. In 2D Laguerre filter, the choice $M=1, N=2$ and $b 1=0.5, b 2=0.5$ leads to 2 free parameters and memory area of 50 , it is obviously a more efficient structure.

\subsection{Adaptive Algorithm}

In the following, we will derive an adaptive algorithm for on-line estimation of the expansion coefficients $w_{k 1 k 2}$. If $d(m, n)$ is the desired response, then the output error will be given by

$$
\begin{aligned}
e(m, n)=d(m, n)-y(m, n) \\
=d(m, n)-\sum_{k 1=0}^{M} \sum_{k 2=0}^{N} w_{k 1 k 2} v_{k 1 k 2}(m, n)
\end{aligned}
$$

In general, the filter parameters are updated by minimizing the mean squared error, i.e.,

$$
J=\frac{1}{2} E\left(e^{2}(m, n)\right)
$$

If steepest descent algorithm is used, then filter parameters can be updated by

$$
w_{k 1 k 2}(j+1)=w_{k 1 k 2}(j)-u \frac{\partial J}{\partial w_{k 1 k 2}}
$$

where iteration number $j=m P+n$ if the size of $x(m, n)$ is $P x P$. It means that the filter parameters are updated line by line. Let us use the instaneous 
value of $J$ to replace its ensemble average value, then we have

$$
w_{k 1 k 2}(j+1)=w_{k 1 k 2}(j)-\frac{u}{2} \frac{\partial e^{2}(m, n)}{\partial w_{k 1 k 2}}
$$

From eq(14), we get

$$
\frac{\partial e^{2}(m, n)}{\partial w_{k 1 k 2}}=-2 e(m, n) v_{k 1 k 2}(m, n)
$$

Thus, adaptive algorithm can be written as

$$
w_{k 1 k 2}(j+1)=w_{k 1 k 2}(j)+u e(m, n) v_{k 1 k 2}(m, n)
$$

The step size $u$ is sufficiently small for ensuring convergence of algorithm.

\subsection{Convergence Condition}

In the following, the stability condition of the mean weight is performed when input signal $x(m, n)$ is white noise.

Fact 3: If $x(m, n)$ is zero mean white noise process with variance $\sigma^{2}$, then it can be shown that

$$
<v_{k 1 k 2}(m, n), v_{k 3 k 4}(m, n)>=\sigma^{2} \delta(k 1-k 3) \delta(k 2-k 4)
$$

Now, taking expected values of both sides of eq(19) and assuming the weight at the iteration number $j$ is independent of $v_{k 1 k 2}(m, n)$, yields a difference equation describing the mean value of the weight:

$$
\begin{aligned}
& E\left(w_{k 1 k 2}(j+1)\right)=\left(1-u \sigma^{2}\right) E\left(w_{k 1 k 2}(j)\right) \\
+ & u E\left(d(m, n) v_{k 1 k 2}(m, n)\right)
\end{aligned}
$$

Note that fact 3 have been used in the above derivation. $\mathrm{Eq}(21)$ converges for large $j$ whenever

$$
\left|1-u \sigma^{2}\right|<1
$$

This condition is equivalent to

$$
0<u<\frac{2}{\sigma^{2}}
$$

Hence, the algorithm converges in the mean sense if step size $u$ is less than the reciprocal of the half of input signal power.

2.6 Example

In this following, we present numerical simulation results when Laguerre filter is used in a $2 \mathrm{D}$ system identification configuration.

The system to be identified is the

$$
A\left(z_{1}, z_{2}\right)=\frac{0.08}{1-0.6 z_{1}^{-1}-0.8 z_{2}^{-1}+0.48 z_{1}^{-1} z_{2}^{-1}}
$$

The input signal $x(m, n)$ is a normal $(0,1)$-distributed white noise with size $30 \times 30$. All computations are performed with Matlab on a IBM PC compatible computer. The parameters used are $M=2, N=2$, and step size $u=0.05$. In order to study the effect of parameters $b 1, b 2$ on the filter performance, we show the relative total error $\beta=E\left(e^{2}(m, n)\right) / E\left(d^{2}(m, n)\right)$ in Fig. 2 after LMS algorithm convergence. It is clear that the $2 \times 2$ D FIR filter $(b 1=0, b 2=0)$ performs poorly $(\beta=0.3502)$ whereas $2 \mathrm{D}$ Laguerre filter with $(b 1=0.5, b 2=0.7)$ performs well $(\beta=0.0006)$. Thus, when the number of free parameters is fixed, $2 \mathrm{D} \mathrm{La}$ guerre filter provides a better modeling characteristics than 2D FIR filter.

\section{Conclusion}

In this paper, a new 2D adaptive Laguerre filter is developed. When long impulse response system is required to identify, computer simulation shows that the 2D Laguerre filter has better performance than the conventional 2D FIR filter at the same computation cost.

\section{References}

[1] J.J.Shynk, "Adaptive IIR filtering", IEEE ASSP Mag., pp.4-21 Apr. 1989

[2] H.Perez and S.Tsujii, "A system identification algorithm using orthogonal functions", IEEE Trans. Signal Processing, Vol.39 pp.752-755 March 1991

[3] B.Wahlberg, "System identification using Laguerre Models", IEEE Trans. Automat. Contr., Vol.36 pp.551-562 May 1991

[4] D.W.Davidson and D.D.Falconer, "Reduced complexity echo cancellation using orthonormal functions", IEEE Trans. Circuit Syst., Vol.38 pp.20-28 Jan. 1991

[5] M.M.Hadhoud and D.W.Thomas, "The two dimensional adaptive LMS (TDLMS) algorithm", IEEE Trans. Circuit Syst., Vol.35 pp.485-494 May 1988

[6] J.C.Principe, B. de Vries, and P.G. de Oliveira,"The gamma filter -a new class of adaptive IIR filters with restricted feedback", IEEE Trans. Signal Processing, Vol.41 pp.649-656 Feb. 1993 


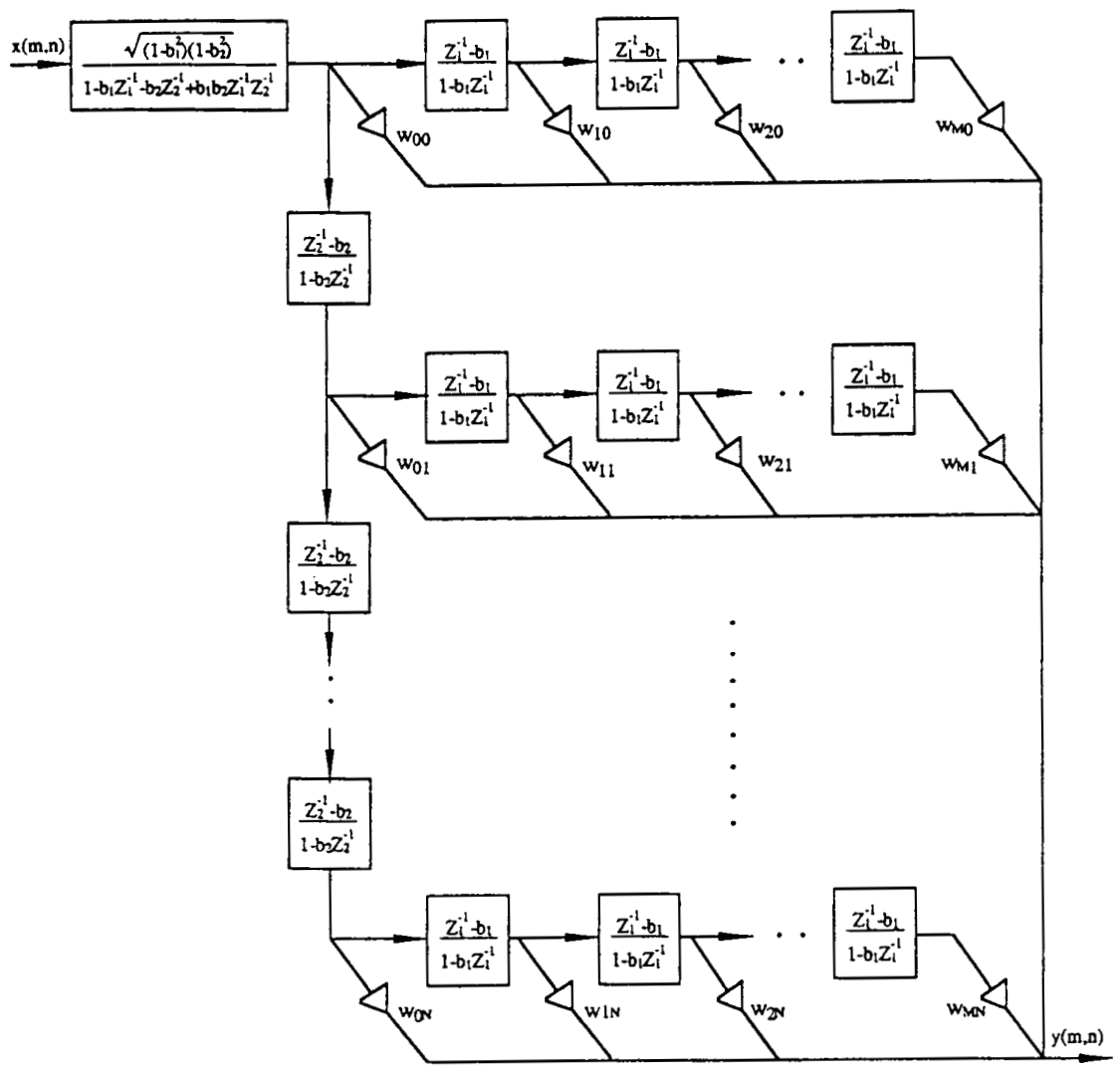

Fig.12D Filter Structure Using Laguerre function.

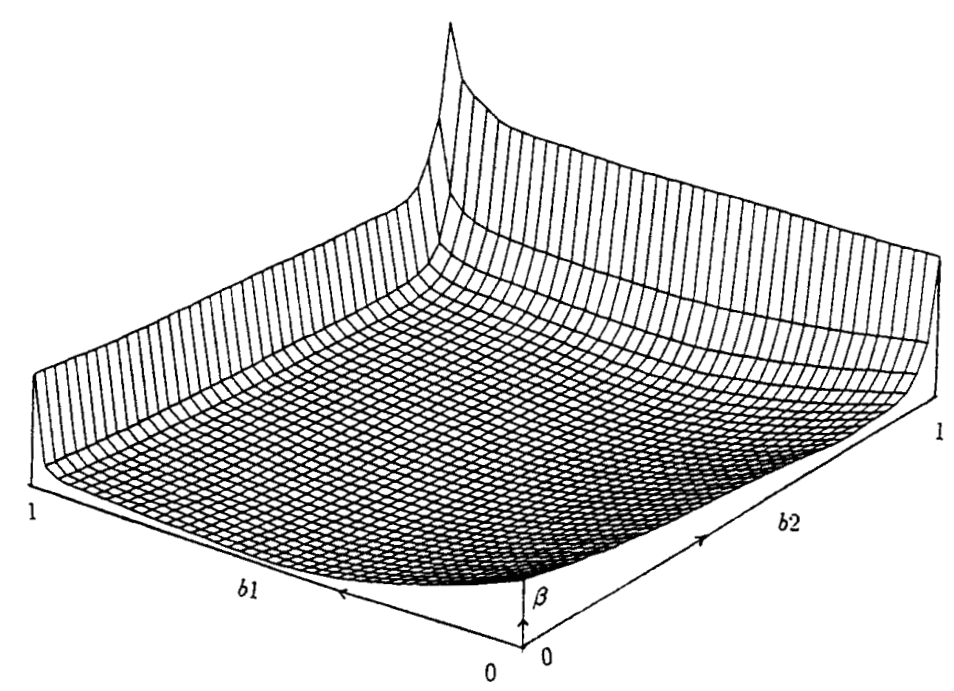

Fig.2 Performance index $\beta$ as a function of $b_{1}, b_{2}$ for IIR system identification. III-420 